\title{
Descripción de Thelastoma domesticus sp. nov. (Oxyurida, Thelastomatidae) parásita de ninfas Periplaneta americana (Blattodea, Blattidae) en Argentina
}

\author{
Nora B. Camino, ${ }^{1,2}$ \& Marco A. Quelas ${ }^{1}$ \\ 1. Centro de Estudios Parasitológicos y de Vectores, CEPAVE, Calle 2 n 584, 1900 La Plata, Argentina. (nemainst@cepave.edu.ar) \\ 2. Investigador CIC.
}

\begin{abstract}
Description of Thelastoma domesticus sp. nov. (Oxyurida, Thelastomatidae) parasite of nymphs of Periplaneta americana (Blattodea, Blattidae) in Argentina. Thelastoma domesticus sp. nov. a parasite of nymphs of Periplaneta americana (Linneus, 1758) found in La Plata, Buenos Aires Province, Argentina is described and illustrated. This new species is characterized by having in the mouth a simple stoma with of thick walls, strongly cuticularised forming three pairs of nodules in two rows, without teeth; the excretory pore is located posterior to the beginning of the intestine, the male presents particular distribution of the genital papillae: a pair of big papillae preanal and three pairs of papillae posanales grouped very close, the second pair of papillae are displased a bit laterally, forming with other posanal papillae a circle.
\end{abstract}

KEYWORDS. Thelastoma domesticus sp. nov., Nematoda, cockroach, taxonomy.

RESUMO. Thelastoma domesticus sp. nov. se halló parasitando a ninfas de Periplaneta americana (Linnaeus, 1758) en la ciudad de La Plata, Província de Buenos Aires, Argentina, en el presente trabajo se describe e ilustra. Esta especie nueva se caracteriza por tener en la boca un estoma simple de paredes gruesas, fuertemente cuticularizada formando tres pares de nodulos en dos filas, sin dientes, el poro excretor es posterior, se ubica al comienzo del intestino, el macho presenta una distribución particular de las papilas genitales: un par grande de papilas preanales y tres pares posanales agrupadas, de las cuales el segundo par se encuentra desplazado lateralmente, configurando así con las otras papilas posanales un círculo.

PALABRAS-CLAVE. Thelastoma domesticus sp. nov., Nematoda, cucaracha, taxonomía.

El estudio de parásitos y patógenos de cucarachas realizado en la zona del Gran La Plata con el objeto de determinar agentes que puedan biocontrolar a esta plaga domiciliaria, reveló la presencia de nemátodos en el tubo digestivo. Estos nemátodos fueron identificados dentro de la familia Thelastomatidae, subfamilia Thelastomatinae, y del género Thelastoma Leidy, 1849. A partir de esa fecha hasta la actualidad el género Thelastoma cuenta con más de 25 especies descriptas en todo el mundo (ADAMSON \& VAN WAEREBEKE, 1992).

En Argentina, en la Provincia de Buenos Aires, se halló dos especies del género: Thelastoma rara Camino et al., 1997 y T. modestus Camino \& Reboredo, 1999, en el intestino de Diloboderus abderus Sturm, 1826 (Coleoptera, Scarabaeidae), relacionados a cultivos de crucíferas. El género caracterizase por tener la boca rodeada de ocho papilas labiales, la cavidad bucal simple, el esófago formado por un pseudobulbo, istmo y bulbo basal valvado, el poro excretor es anterior a la base del esófago, el apéndice caudal de la hembra es filiforme, la vulva se ubica en la zona media del cuerpo, didélfica, el apéndice caudal del macho es alargado, con cuatro pares de papilas genitales.

En el presente trabajo se describe a una especie nueva del género Thelastoma parásita de la cucaracha Periplaneta americana (Linnaeus, 1758) (Blattodea, Blattidae).

\section{MATERIALES Y METODOS}

En una casa ubicada en el barrio norte de la ciudad de La Plata (34 $55^{\circ}$ S ; 5 $\left.57^{\circ} 57^{\prime} \mathrm{O}\right)$, Provincia de Buenos Aires,
Argentina, se colectaron manualmente ninfas de $P$. americana $(\mathrm{n}=26)$. Según POINAR (1975) las ninfas de cucaracha fueron colocadas en un recipiente de plástico cubiertas con tierra del lugar. En el laboratorio fueron anestesiadas con frío $5^{\circ} \mathrm{C}$ durante 3 minutos y luego disectadas bajo microscopio estreoscópico en cápsulas de Petri con agua destilada. Los nemátodos fueron hallados vivos en el proctodeo intestinal, encontrándose machos, hembras y larvas. Para su estudio taxonómico se los mató en agua destilada a $60^{\circ} \mathrm{C}$ durante 2 minutos; posteriormente fueron colocados durante 48 horas en una solución de partes iguales de agua destilada y TAF (formol $7 \mathrm{ml}$, triethanolamina $2 \mathrm{ml}$ y agua destilada $91 \mathrm{ml}$ ), para luego terminar la fijación en TAF puro. En un microscópio óptico con cámara clara fueron obtenidas las medidas y realizados los dibujos. Los paratipos se encuentran depositados en el Departamento Científico del Centro de Estudios Parasitológicos y de Vectores, CEPAVE, y los holotipo y alotipo se depositaran en la colección Helmintologica del Museo de La Plata, La Plata, Argentina.

\section{RESULTADOS}

Thelastoma domesticus sp. nov. (Figs.1-11)

Diagnosis. Cavidad bucal simple de paredes gruesas, fuertemente cuticularizadas formando tres pares de nódulos en dos filas, sin dientes; el poro excretor se ubica muy posterior al comienzo del intestino; el macho 


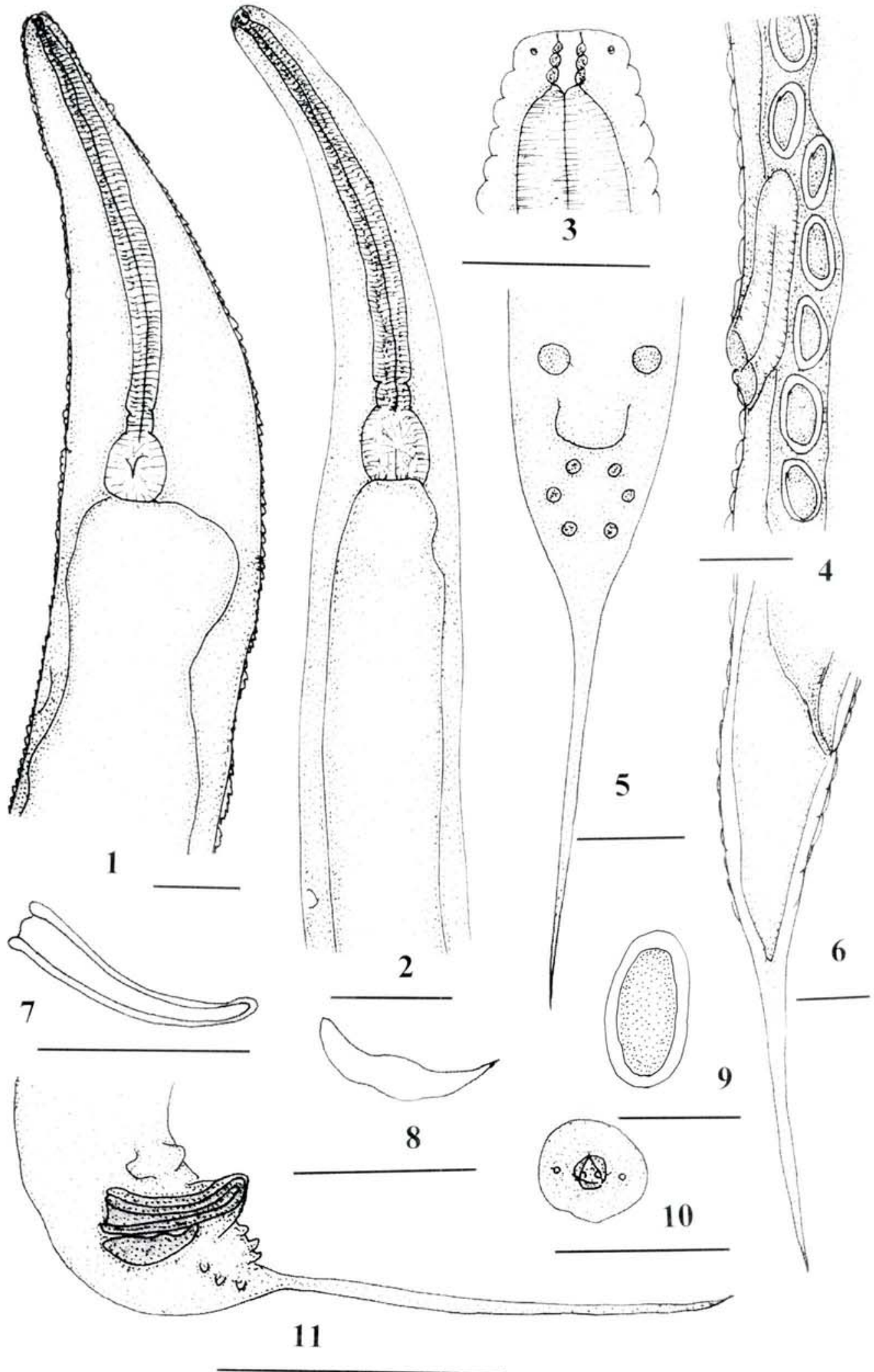

Figs. 1-11, Thelastoma domesticus sp. nov. 1, extremo anterior de la hembra; 2, extremo anterior del macho; 3, estoma de la hembra; 4, vagina y úteros con huevos; 5 , extremo caudal del macho en vista ventral; 6, extremo caudal de la hembra; 7, espícula; 8, gubernáculo; 9, huevo; 10, boca de la hembra en vista apical; 11, extremo caudal del macho en vista lateral. (Barras: 1, 2, 4, 9, 11=50 $10=25 \mu \mathrm{m})$. 
presenta una disposición propia de las papilas genitales de manera siguiente: un par de papilas grandes preanales y tres pares de papilas posanales agrupadas muy próximas, el segundo par de papilas se hallan desplazadas un poco lateralmente.

Descripción. Cuerpo de la hembra fusiforme, bastante grueso. Boca rodeada de ocho papilas labiales. Anfidios pequeños y piriformes. Cavidad bucal con un estoma simple de paredes gruesas, fuertemente cuticularizadas, formando tres pares de nódulos en dos filas, sin dientes (Fig. 3); la abertura bucal triangular configurando tres labios iguales (Fig. 10). Cutícula anillada a lo largo de todo el cuerpo, diferenciándose el primer anillo que es más ancho, el resto de los anillos son finos y de igual tamaño. Alas laterales ausentes. Esófago largo presentando un pseudobulbo largo no valvado y un bulbo basal con valvas, ambos separados por un istmo corto (Fig. 1). Poro excretor muy posterior al comienzo del intestino. Vulva protuberante, ubicada en la parte media posterior del cuerpo. Vagina larga, musculosa, paralela al eje longitudinal del cuerpo y dividida en dos porciones: la vagina muscular y la vagina vera (Fig. 4), úteros y oviductos opuestos contienen los huevos, y están presentes los dos ovarios. Huevos con cáscara lisa, sin ornamentaciones, dispuestos individualmente de manera desordenada, y no se encuentran embrionados (Figs. 4, 9). Apéndice caudal lagro, fuerte y filiforme (Fig. 6). Macho muy pequeño respecto al tamaño de la hembra. Cutícula suavemente anillada a lo largo de todo el cuerpo, siendo todos los anillos finos e iguales en tamaño. Anfidios visibles como pequeños puntos. Cavidad bucal simple, sin dientes y de sección triangular. Esófago igual al de la hembra, pero de una longitud más corta (Fig. 2). Poro excretor muy posterior al comienzo del intestino. Espícula simple, gruesa, de puntera redondeada, sin ornamentaciones (Fig. 7). Gubernáculo más corto que la espícula, grueso y sin proyecciones (Fig. 8). Cono genital bien desarrollado (Fig. 11). Papilas genitales en cuatro pares dispuestas de la siguiente manera: un par de papilas grandes preanales y tres pares de papilas posanales agrupadas muy próximas, el segundo par de papilas se hallan desplazadas un poco lateralmente (Fig. 5). Apéndice caudal largo y filiforme.

Macho (n=10). Longitud total: $990 \mu \mathrm{m}$ (860-990); diámetro de la cabeza a nivel de las papilas cefálicas: 12 $\mu \mathrm{m}(11,75-14,1)$; diámetro del cuerpo a nivel del anillo nervioso: $47 \mu \mathrm{m}$ (46-62); diámetro máximo del cuerpo: 68 $\mu \mathrm{m}(65,8-70,5)$; diámetro del cuerpo a nivel del ano: 72,8 $\mu \mathrm{m}$ (70,5-82); longitud del esófago: 360 um (320-380); distancia del extremo anterior al poro excretor: $380 \mu \mathrm{m}$ (360-440); longitud de las espículas: 28,2 $\mu \mathrm{m}$ (25,85-39,95); longitud del gubernaculo: $22,32 \mu \mathrm{m}(18,8-23,5)$; longitud del apéndice caudal: $82 \mu \mathrm{m}(81,25-84,6)$.

Hembra ( $n=12)$. Longitud total: $2.550 \mu \mathrm{m}$ (2.3103.100); diámetro de la cabeza a nivel de las papilas cefálicas: $16 \mu \mathrm{m}(115,45-18,8)$; diámetro del cuerpo a nivel del anillo nervioso: $64 \mu \mathrm{m}$ (57-68); diámtero máximo del cuerpo: 101,15 $\mu \mathrm{m}(98,7-105,75)$; diámetro del cuerpo a nivel del ano: 94 m (76-98,7); diámetro del cuerpo a nivel de la vulva: $108 \mu \mathrm{m}$ (105-112); longitud del esófago: 390 $\mu \mathrm{m}$ (380-410); distancia del extremo anterior al poro excretor: $400 \mu \mathrm{m}$ (380-480); longitud de la vagina: $148 \mu \mathrm{m}$ (144-156); ancho de la vagina: $48 \mu \mathrm{m}$ (48-52); V: $52 \%$ (51$54)$; longitud y diámetro de los huevos: $68,15 \mu \mathrm{m}(63,45-$ 70,5) x 33,84 $\mu \mathrm{m}(32,9-35,25)$; longitud del apéndice caudal: $108,1 \mu \mathrm{m}(105-110,45)$.

Hospedador tipo. Ninfas de Periplaneta americana (Blattodea, Blattidae).

Localidad tipo. Casa particular, barrio norte, ciudad de La Plata (34 $55^{\circ}$ S ; 5 5 $57^{\circ}$ 'O), Provincia de Buenos Aires, Argentina. Colectado por los autores en septiembre de 2005.

\section{DISCUSION}

Thelastoma domesticus sp. nov. es semejante a las siguientes especies: T. atternuatum Leidy, 1853; $T$. dessetae Adamson, 1985; T. icemi Travassos, 1929; T. macramphidium Christie, 1931; T. modestus Camino y Reboredo, 1999; T. nasuta Kloss, 1965; T. paronai Kloss, 1965; T. periplaneticola Leibersperger, 1960; T. rara Camino et al., 1997 y T. robustum Leidy, 1853 compartiendo la posición del poro excretor que es anterior al bulbo basal, la vulva no es protuberante y las hembras son muy grandes en tamaño respecto de los machos. Thelastoma atternuatum se caracteriza por el macho sin papila preanal. Thelastoma dessetae no se conocen los machos pero se separa por que poseen un receptáculo seminal en las hembras ubicado posteriormente. Thelastoma icemi se separa por las hembras con un apéndice caudal de un longitud que equivale a 1/6 del largo del cuerpo y por el número de papilas genitales que se encuentran en un par preanal y uno posanal. Thelastoma macramphidium se aleja por poseer dos pares de papilas preanales, un par posanal y un par en la mitad de la longitud del apéndice caudal. Thelastoma modestus se caracteriza por poseer los machos tres pares de papilas posanales de los cuales el último se ubica en la base del apéndice caudal. Thelastoma nasuta se aleja por poseer la región vulvar muy saliente y fuertemente grueso formando una saliencia en forma de nariz y en los machos la abertura año-genital se encuentra rodeada de grandes papilas y un par se ubica en el medio del apéndice caudal. Thelastoma paronai se separa por la hembra poseer alas laterales que se presentan desde el anillo nervioso hasta casi la región anal y el macho cuenta con una papila caudal próxima al inicio del apéndice caudal. Thelastoma periplaneticola se diferencia de Thelastoma domesticus sp. nov. en la disposición de las papilas genitales del macho, que posee dos pares de papilas preanales ubicadas en un promontorio donde también se encuentran un par adanal y la existencia de un par situado en la mitad del apéndice caudal. Thelastoma rara se caracteriza por las once papilas genitales masculinas, una simple y grande papilas preanal, cuatro pares posanales dispuestas el primer par ventral, el segundo par lateral respecto al anterior, el tercer par ventrolateral y el cuarto par ventral, esta cuatro últimas papilas se hallan a la misma 
altura y en la base del apéndice caudal. Thelastoma robustum puede diferenciarse de T. domesticus sp. nov. en que la hembra posee un apéndice caudal de una longitud igual a 1/3 del largo corporal y por el número y disposición de papilas posanales, las cuales se presentan en seis pares, estando el segundo par fusionado.

Por estas diferencias taxonómicas es que consideramos a $T$. domesticus sp. nov. como una nueva especie para la ciencia, caracterizándose principalmente por la disposición de las papilas genitales del macho en un par de grandes papilas preanales y tres pares posanales agrupadas constituyendo un círculo.

\section{REFERENCIAS BIBLIOGRAFICAS}

Adamson, M. L. \& van Waerebeke, D. 1992. Revision of Thelastomatoidea, Oxyurida of invertebrate sosts I. Thelastomatidae. Systematic Parasitology 21:21-63.

PoINAR, G. O. JR. 1975. Entomogenous nematodes. A manual and host list of insect nematode associations. Leiden, E. J. Brill. $317 \mathrm{p}$. 